\title{
Operations and Actions of Lie Groups on Manifolds
}

\author{
Sharmin Akter ${ }^{1}$, Mir Md. Moheuddin ${ }^{1}$, Saddam Hossain ${ }^{2}$, Asia Khatun ${ }^{1}$ \\ ${ }^{1}$ Department of CSE (Mathematics), Atish Dipankar University of Science and Technology (ADUST), Dhaka, Bangladesh \\ ${ }^{2}$ Department of Basic Science (Mathematics), World University of Bangladesh (WUB), Dhaka, Bangladesh \\ Email: Sharmindu2005@gmail.com,mirmdmoheuddinkhan@gmail.com, s.hossain656@gmail.com, asiakhatun33@yahoo.com
}

How to cite this paper: Akter, S., Moheuddin, M.Md., Hossain, S. and Khatun, A. (2020) Operations and Actions of Lie Groups on Manifolds. American Journal of Computational Mathematics, 10, 460-472. https://doi.org/10.4236/ajcm.2020.103026

Received: July 2, 2020

Accepted: September 24, 2020

Published: September 27, 2020

Copyright (c) 2020 by author(s) and Scientific Research Publishing Inc. This work is licensed under the Creative Commons Attribution International License (CC BY 4.0).

http://creativecommons.org/licenses/by/4.0/ (c) (i) Open Access

\begin{abstract}
As recounted in this paper, the idea of groups is one that has evolved from some very intuitive concepts. We can do binary operations like adding or multiplying two elements and also binary operations like taking the square root of an element (in this case the result is not always in the set). In this paper, we aim to find the operations and actions of Lie groups on manifolds. These actions can be applied to the matrix group and Bi-invariant forms of Lie groups and to generalize the eigenvalues and eigenfunctions of differential operators on $\mathbb{R}^{n}$. A Lie group is a group as well as differentiable manifold, with the property that the group operations are compatible with the smooth structure on which group manipulations, product and inverse, are distinct. It plays an extremely important role in the theory of fiber bundles and also finds vast applications in physics. It represents the best-developed theory of continuous symmetry of mathematical objects and structures, which makes them indispensable tools for many parts of contemporary mathematics, as well as for modern theoretical physics. Here we did work flat out to represent the mathematical aspects of Lie groups on manifolds.
\end{abstract}

\section{Keywords}

Group $(G)$, Abelian Group $\left(g_{1} g_{2}=g_{2} g_{1}\right)$, Subgroup ( $H$ Is a Subgroup of $G$ ),

Co-Sets $(g H)$, Lie Groups $\left(G \times G \rightarrow G(x, y) \mapsto x \cdot y\right.$ and $\left.G \rightarrow G, g \mapsto g^{-1}\right)$, Smooth Mapping $(\sigma: G \times G \rightarrow G)$

\section{Introduction}

In the present era, the study of the group related to the Lie group is essential for the sake of its comprehensive applications in several fields. Through the mathematical analysis, representations of groups on the manifold are vital due to it al- 
lows many group-theoretic problems in form of linear algebra problems, which is well perceived. However, Lie groups were significantly studied by Marius Sophus Lie (1842-1899), who manipulated to solve ordinary differential equations. Lie groups and Lie algebras, together with acquainted Lie theory which plays an effective role in the branch of pure and applied mathematics that is utilized in modern physics as well as an active area of research.

By the literature view, we can observe that many researchers have endeavored to analyze and discuss the importance of operations and actions of Lie groups on manifolds in various fields. In [1], Nahm et al. defined strictly semi-simple graded Lie algebras and depicted to have properties similar to semi-simple Lie algebras, presented their classification. Cahen et al. [2], proved the existence of a * product on the cotangent bundle of a parallelizable manifold $M$. Besides, Gelfand et al. [3], presented some important nonlocal representations of the group Gx consisting of C-mappings of a Riemannian Manifold X to a compact semisimple Lie Group G are constructed. The irreducibility, as well as non-equivalence of the introduced representations corresponding to different Riemannian metrics, is also proved in their paper. Moreover, in [4], Albeverio et al. constructed a unitary representation of the Sobolev-Lie group of $\mathrm{C}$, mappings from an orientable Riemann manifold M to a Lie Group $\mathrm{G}$ with compact Lie algebra. The representation is given in terms of the energy function on $\mathrm{Cj}(\mathrm{M}, \mathrm{G})$ and provides a new type of representations of current algebras. Celledoni et al. [5] showed how the methods look when applied to the rigid body equations in particular and indicated how the methods work in general. In their paper, they also discussed how the computation of these maps can be optimized for the rigid body case, and provided numerical experiments which give an idea of the performance of Lie group methods compared to other known integration schemes. In [6], Gepner et al. addressed a number of issues concerning affine Lie algebras and string propagation on group manifolds. They also showed that a $1+1$ dimensional quantum field theory which gives a realization of current algebra. Besides, Iserles et al. [7] represented the solution as an infinite series whose terms are indexed by binary trees, by building upon an earlier work of Wilhelm Magnus. In [8], Goldman et al. studied one large class of groups: fundamental groups of compact Kahler manifolds; and deduced a general local property of their varieties of representations near representations satisfying fairly general conditions. What is more, Iserles et al. [9] surveyed the novel theory of numerical integrators that respect Lie-group structure, highlighting theory, algorithmic issues and various applications. In [10], Jean Gallier et al. introduced a framework based on Riemannian Geometry for defining some basic statistical notions on curved spaces and to make a relation between Lie groups and manifolds. Also, Neeb et al. [11] reported on the state of the art of some of the fundamental problems in the Lie theory of Lie groups modelled on locally convex spaces, such as integrability of Lie algebras, integrability of Lie subalgebras to Lie subgroups, and integrability of Lie algebra extensions to Lie group extensions. In [12], Ferreira et al. presented lecture notes based on elements of group theory, lie groups and Lie alge- 
bras, representation theory of Lie algebras.

In the present tasks, we discussed the Group and Abelian groups also Subgroups with the Lie groups based on the smooth manifolds that assist us to understand the properties and actions of the Lie groups on Manifolds. We also reveal some example which shows a clear view of operations and actions of Lie groups on Manifolds.

The paper is organized as follows: in Sections 2 and 3, we introduce an overview of the group, Abelian group and the Lie group that are used in the next Section. Section 4 shows smooth mapping on Lie group with the manifold. In Section 5 includes a conclusion and recommendations of our works.

\section{Group}

\subsection{Introduction}

Group theory studies the algebraic structures known as groups. The concept of a group is central to abstract algebra: other well-known algebraic structures, such as rings, fields, and vector spaces.

Agroup is a set $G$ equipped with a binary operation: $G \times G \rightarrow G$ that associates an element $a \cdot b \in G$ to every pair of elements $a, b \in G$, and having the following properties:- is associative, has an identity element $e \in G$, and every element in $G$ is invertible (w.r.t.). More explicitly, this means that the following equations hold for all $a, b, c \in G$ :

(G1) $a \cdot(b \cdot c)=(a \cdot b) \cdot c \cdot$ (associativity).

(G2) $a \cdot e=e \cdot a=a$. (identity).

(G3) For every $a \in G$, there is some $a^{-1} \in G$ such that $a \cdot a^{-1}=a^{-1} \cdot a=e$. (inverse).

Otherwise, we say two elements, $g_{1}$ and $g_{2}$ of a group commute with each other if their product is independent of the order, i.e., if $g_{1} g_{2}=g_{2} g_{1}$. If all elements of a given group commute with one another then we say that this group is Abelian. The real numbers under addition or multiplication (without zero) form an Abelian group. The cyclic groups $Z_{n}$ are Abelian for any $n$. The symmetric group $S_{n}$ is not Abelian for $n>2$, but it is Abelian for $n=2$. Simplexes are building blocks of a polyhedron. As shown in Figure 1, a 0 -simplex $\left\langle P_{0}\right\rangle$ is a point or a vertex, and a 1-simplex $\left\langle P_{0} P_{1}\right\rangle$ is a line or an edge. A 2-simplex $\left\langle P_{0} P_{1} P_{2}\right\rangle$ is defined to be a triangle with its interior included and a 3-simplex $\left\langle P_{0} P_{1} P_{2} P_{3}\right\rangle$ is a solid tetrahedron.

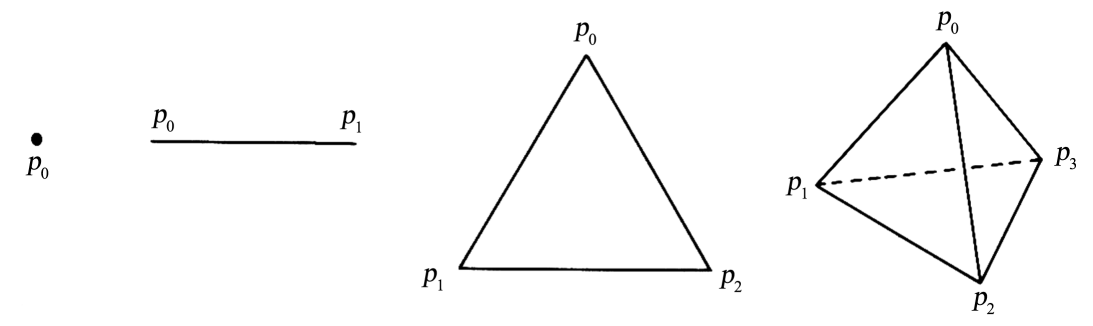

Figure 1. The elements of $S_{2}$. 


\subsection{Example of Group}

Let us consider some groups of order two, i.e., with two elements. The elements 0 and 1 form a group under addition modulo 2 . We have

$$
0+0=0 ; 0+1=1 ; 1+0=1 ; 1+1=0
$$

The elements 1 and -1 also form a group but under multiplication. We have

$$
1 \cdot 1=-1 \cdot(-1)=1 ; 1 \cdot(-1)=(-1) \cdot 1=-1
$$

The symmetric group of degree 2, $S_{2}$ has two which is shown in Figure 2.

That satisfies

$$
e \cdot e=e, e \cdot a=a \cdot e=a, a \cdot a=e
$$

these three examples of groups are different realizations of the same abstract group. If we make the identifications as shown above. We see that the structure of these groups is the same.

In Figure 3, we say that these groups are isomorphic.

\subsection{Isomorphism and Homomorphism of Group}

\section{Isomorphism 2.3.1.}

Two groups $G$ and $G^{\prime}$ are isomorphic if their elements can be put into a one-to-one correspondence which is preserved under the composition laws of the groups. The mapping between these two groups is called an isomorphism.

Homomorphism 2.3.2.

A group $G$ being mapped into another group $G^{\prime}$ but not in a one-to-one manner, i.e. two or more elements of $G$ are mapped into just one element of $G^{\prime}$. If such mapping respects the product law of the groups we say they are homomorphic. The mapping is then called a homomorphism between $G$ and $G^{\prime}$.

From Figure 4, let $G$ and $G^{\prime}$ be Abelian groups. A map $f: G \rightarrow G^{\prime}$ is said to be a homomorphism if

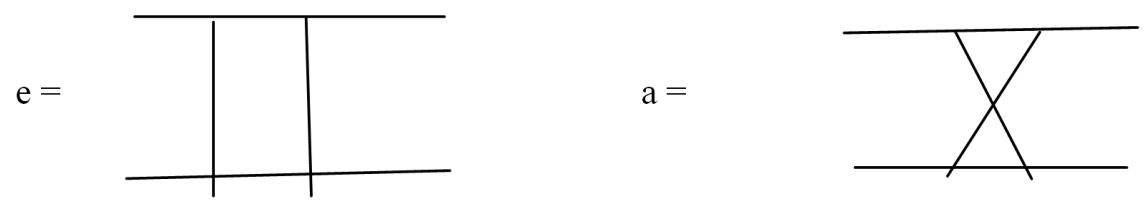

Figure 2. The elements of $S_{2}$.

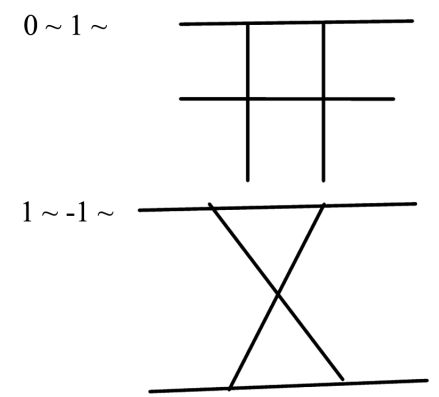

Figure 3. Isomorphism. 


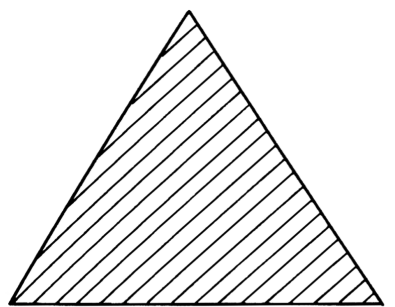

(a)

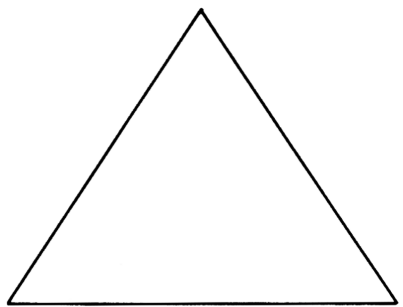

(b)

Figure 4. (a) is a solid triangle while and (b) is the edges of a triangle without an interior.

$$
f(x+y)=f(x)+f(y)
$$

Example: Consider the cyclic groups $Z_{6}$ with elements $e, a, a^{2}, \cdots, a^{5}$ and $a^{6}=e$, and $Z_{6}$ with elements $e^{\prime}$ and $b\left(b^{2}=e^{\prime}\right)$. The mapping $\sigma: Z_{6} \rightarrow Z_{2}$ given by

$$
\begin{aligned}
& \sigma(e)=\sigma\left(a^{2}\right)=\sigma\left(a^{4}\right)=e^{\prime} \\
& \sigma(e)=\sigma\left(a^{2}\right)=\sigma\left(a^{4}\right)=b
\end{aligned}
$$

is a homomorphism between $Z_{6}$ and $Z_{2}$.

\subsection{Subgroup}

\section{Definition 2.4.1.}

Given a group $G$, a subset $H$ of $G$ is a subgroup of $G$ iff

1) The identity element $e$ of $G$ also belongs to $H(e \in H)$;

2) For all $h_{1}, h_{2} \in H$, we have $h_{1} h_{2} \in H$;

3) For all $h \in H$, we have $h^{-1} \in H$.

It is easily checked that a subset $H \subseteq G$ is a subgroup of $G$ iff $H$ is nonempty and whenever $h_{1}, h_{2} \in H$, then $h_{1} h_{2}^{-1} \in H$.

\section{Definition 2.4.2.}

A subset $H$ of a group $G$ which satisfies the group postulates under the same composition law used for $G$, is said to be a subgroup of $G$. The identity element and the whole group $G$ itself are subgroups of $G$. They are called improper subgroups. All other subgroups of a group $G$ are called proper subgroups. If $H$ is a subgroup of $G$, and $K$ a subgroup of $H$, then $K$ is a subgroup of $G$.

\subsection{Cosets}

If $H$ is a subgroup of $G$ and $g \in G$ is any element, the sets of the form $g H$ are called left cosets of $H$ in $G$ and the sets of the form $H g$ are called right cosets of $H$ in $G$.

Left cosets (resp. right cosets) of $H$ induce an equivalence relation defined as follows:

For all $g_{1}, g_{2} \in G, g_{1} \sim g_{2}$ iff $g_{1} H=g_{2} H \quad$ (resp. $g_{1} \sim g_{2}$ iff $H g_{1}=H g_{2}$ Obviously, is an equivalence relation.

Given a group $G$ and a subgroup $H$ of $G$ we can divide the group $G$ into disjoint sets such that any two elements of a given set differ by an element of $H$ 
multiplied from the right. That is, we construct the sets $g H \equiv\{$ All elements $g h$ of $G$ such that $h$ is any element of $H$ and $g$ is a fixed element of $G$.

\subsection{Theorem}

The order of a subgroup of a finite group is a divisor of the order of the group.

Proof. For a finite group, $G$ of order $m$ with a proper subgroup $H$ of order $n$, we can write

$$
m=k n
$$

where $k$ is the number of disjoint sets $g H$.

The set of elements $g H$ are called left cosets of $H$ in $G$. They are certainly not subgroups of $G$ since they do not contain the identity element, except for the set $e H=H$. Analogously we could have split $G$ into sets $H g$ which are formed by elements of $G$ which differ by an element of $H$ multiplied from the left. The same results would be true for these sets. They are called right cosets of $H$ in $G$. The set of left cosets of $H$ in $G$ is denoted by $G / H$ and is called the left coset space. An element of $G / H$ is a set of elements of $G$, namely $g H$.

Analogously the set of right cosets of $H$ in $G$ is denoted by H/G and it is called the right coset space. If the subgroup $H$ of $G$ is an invariant subgroup, then the left and right cosets are the same since $g^{-1} \mathrm{Hg}=H$ implies $g H=H g$. In addition, the coset space $G / H$, for the case in which $H$ is invariant, has the structure of a group and it is called the factor group or the quotient group. To show this, we consider the product of two elements of two different cosets. We get

$$
g h_{1} g^{\prime} h_{2}=g g^{\prime} g^{\prime-1} h_{1} g^{\prime} h_{2}=g g^{-1} h_{3} h_{2}
$$

where we have used the fact that $H$ is invariant, and therefore there exists $h_{3} \in H$ such that $g^{\prime-1} h_{1} g^{\prime}=h_{3}$. Thus, we have obtained an element of a third coset, namely $g g^{\prime} H$. If we had taken any other elements of the cosets $g H$ and $g^{\prime} H$, their product would produce an element of the same coset $g g^{\prime} H$. Consequently, we can introduce, in a well-defined way, the product of elements of the coset space $G / H$, namely

$$
g H g^{\prime} H \equiv g g^{\prime} H
$$

The invariant subgroup $H$ plays the role of the identity element since

$$
(g H) H=H(g H)=g H
$$

The inverse element is $g^{-1} H$ since

$$
g^{\prime} H g H=g^{-1} g H=H=g H g^{-1} H
$$

The associatively is guaranteed by the associatively of the composition law of the group $G$. Therefore, the coset space $G / H$ and $H / G$ is a group in the case, where $H$ is an invariant subgroup. Such group is not necessarily a subgroup of $G$ or $H$.

This completes the proof of the theorem.

\section{Lie Groups}

Let $G$ is a differentiable manifold which is endowed with a group structure such 
that the group operations.

1) $G \times G \rightarrow G,\left(g_{1}, g_{2}\right) \rightarrow g_{1} \cdot g_{2}$

2) $G \rightarrow G, g \mapsto g^{-1}$

are differentiable. It can be shown that $G$ has a unique analytic structure with which the product and the inverse operations are written as a convergent power series.

The unit element of a Lie group is written as $e$. The dimension of a Lie Group $G$ is defined to be the dimension of $G$ of a manifold. The product symbol may be omitted and $g_{1} \cdot g_{2}$ is usually written as $g_{1} g_{2}$. If the product is commutative, namely $g_{1} \cdot g_{2}=g_{1} \cdot g_{2}$, we often use the additive symbol + instead of the product symbol.

\subsection{Some Basic Definitions of Lie Groups}

\section{Definition 3.1.1}

A Lie Group $G$ is a group and a smooth manifold such that group multiplication $G \times G \rightarrow G(x, y) \mapsto x \cdot y$ and group inversion $G \rightarrow G x \mapsto x^{-1}$ are smooth maps. As shown in Figure 5, if $G$ and $H$ are Lie groups, a Lie group homomorphism $\varphi: G \rightarrow H$ is a smooth mapping which is also a homeomorphism of the abstract groups. If the mapping is a diffeomorphism, then $\varphi$ is called an isomorphism. Much of the structure of Lie groups comes from the so-called left and right translations. Such as

\section{Definition 3.1.2}

Let $G$ be a Lie group, $s \in G$. The left translation by $s$ is the map $L_{s}: G \rightarrow G$ is given by $L_{s}(t)=S t$ for every $s \in G$. Right translations are defined analogously. The group structure implies that for every $s, L_{s}$ and $R_{s}$ are bijections with inverses $L_{s}^{-1}$ and $R_{s}^{-1}$. The conditions in definition 1 imply that both of these maps (and their inverses) are smooth. Thus, left and right translations are diffeomorphisms of $G$ onto itself.

Familiar examples of Lie groups include $\mathbb{R}^{n}$ under addition and the elementary matrix groups $(G L(n, \mathbb{R}), S L(n, \mathbb{R}), O(n), U(n)$, etc.). In fact, all of the matrix groups can be viewed as closed Lie subgroups of $G L(n, C)$. In particular, the circle group $S^{1} \cong U(1)$ is a Lie group. The product of Lie groups given the standard differentiable and group structures is again a Lie group.

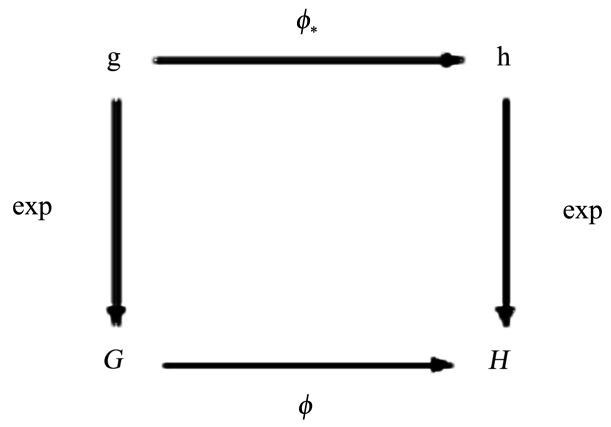

Figure 5. A lie group homomorphism. 
A real Lie group is called a compact Lie group (or connected, simply connected Lie group, etc) if its underlying topological space is compact (or connected, simply connected, etc.).

\subsection{Some Basic Examples of Lie Groups}

1) The real line $\mathbb{R}$ with its standard smooth structure and the group operation being addition is a Lie group. So is every Cartesian space $\mathbb{R}^{n}$ with the component wise addition of real numbers.

2) The quotient of $\mathbb{R}$ by the subgroup of integers $\mathbb{Z} \rightarrow \mathbb{R}$ is the circle group $S^{1}=\mathbb{R} / \mathbb{Z}$. The quotient $\mathbb{R}^{n} / \mathbb{Z}^{n}$ is $n$-dimensional Torus.

3) The automorphism group of any Lie group is canonically itself a Lie group.

4) The general linear group $G L(n)$.

5) The orthogonal group $O(n)$ and special orthogonal group $S O(n)$.

6) The unitary group $U(n)$ and special unitary group $S U(n)$.

7) The symplectic group $S P(2 n)$.

\subsection{Theorem}

The Special Linear group $S L(n)$ is a Lie Group.

Proof: Let $f: G L(n) \rightarrow \mathbb{R}$ is given by $f(A)=\operatorname{det}(A)$ is differentiable. The level set $f^{-1}(1)$ is given by,

$$
S L(n)=\left\{A \in M_{n \times n} / \operatorname{det}(A)=1\right\}
$$

The Special linear group. The derivative of $f$ is surjective at a point $A \in G L(n)$, making $S L(n)$ into a Lie group. Such that

$$
(d f)_{I}(B)=\lim _{h \rightarrow 0} \frac{\operatorname{det}(I+h B)-\operatorname{det}(I)}{h}=\operatorname{tr}(B)
$$

implying that

$$
\begin{aligned}
(d f)_{A}(B) & =\lim _{h \rightarrow 0} \frac{\operatorname{det}(A+h B)-\operatorname{det}(A)}{h} \\
& =\lim _{h \rightarrow 0} \frac{\operatorname{det}(A) \operatorname{det}\left(I+h A^{-1} B\right)-\operatorname{det}(A)}{h} \\
& =\operatorname{det}(A) \lim _{h \rightarrow 0} \frac{\operatorname{det}\left(I+h A^{-1} B\right)-1}{h} \\
& =\operatorname{det}(A)(d f)_{I}\left(A^{-1} B\right) \\
& =\operatorname{det}(A) \operatorname{tr}\left(A^{-1} B\right)
\end{aligned}
$$

since $\operatorname{det}(A)=1$ for any $k \in R$. We can take the matrix $B=\frac{k}{N} A$ to obtain $(d f)_{A}(B)=\operatorname{tr}\left(\frac{k}{N} I\right)=K$, therefore $(d f)_{A}$ is surjective for every $A \in S L(n)$.

Consequently, $S L(n)$ is a sub manifold of $G L(n)$. Therefore, the group multiplication and inversion are differentiable, so $S L(n)$ is a Lie Group. 


\section{Smooth Mapping on Lie Group}

\subsection{Definition}

A Lie group is a smooth manifold whose underlying set of elements is equipped with the structure of a group such that the group multiplication and inverse-assigning functions are smooth functions. The first order infinitesimal approximation to a Lie group is its Lie algebra.

By Figure 6, it is obvious that a Lie group is a smooth manifold such that every connected finite-dimensional real Lie group is homeomorphic to a product of a compact Lie group (its maximal compact subgroup) and a Euclidean space.

\subsection{Operations of Lie Group on the Manifold}

We suppose that this Lie group operation which may be considered as a mapping

$$
G \times G \rightarrow G
$$

is smooth and also that the map $x \rightarrow x^{-1}$ on $G \rightarrow G$ is smooth.

With each element $x$ in $G$ there is associated a transformation $L_{x}$ of $G$ called left translation:

$$
L_{x}^{*} \omega=\omega \text { for all } x \text { in } G .
$$

Let denote the unit element of $G$. the left translation $L_{x}^{-1}=L_{x^{-1}}$ sends $x$ to $e$. If $\omega$ is left invariant, $\omega=L_{x^{-1}}^{*} \omega$ is completely determined at $x$ by its value $\omega_{0}$ at $e$. If $\omega_{0}$ is any given $p$-form ate then a left invariant form $\omega$ is defined by

$$
\omega_{x}=L_{x^{-1}}^{*} \omega_{0}
$$

Let $n$ be the dimension of $G$. Since the space of one form at $e$ is an $n$-dimensional linear space, there are exactly $n$ linearly independent left invariant one forms on $G$. Let

$$
\sigma^{1}, \cdots, \sigma^{n}
$$

be such a system. Any other left invariant one form is a linear combination of these with constant coefficients.

More generally, if $\omega$ is any left invariant $p$-form on $G$,

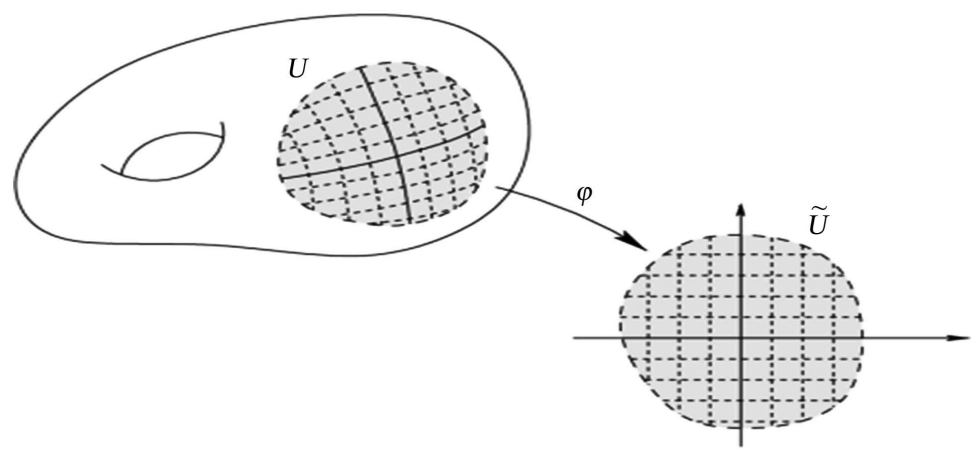

Figure 6. Smooth mapping on lie group. 


$$
\omega=\sum c_{H} \sigma^{H}
$$

where the $c_{H}$ are constants and $\sigma^{H}=\sigma^{h_{1}} \cdots \sigma^{h_{p}}$. Any $p$-form $\omega$ can be expanded in this way and the coefficients $c_{H}$ will in general be scalars on $G$, supposing $\omega$ left invariant forces each of these scalars to be left invariant. This means that each $c_{H}$ takes the same value at each point of $G$, hence is constant.

\subsection{Example}

We shall determine the local structure of all one-dimensional groups.

Solution: Let $t$ be a parameter on $G$, chosen so that $t=0$ is the identity $e$. Let $\sigma$ be a nontrivial left invariant one form; locally,

$$
\sigma=f(t) \mathrm{d} t \text { never zero. }
$$

We integrate $\sigma$ to get a new parameter for $G$,

$$
\int_{0}^{t} f(t) \mathrm{d} t
$$

thus, we may assume we have started with a parameterization of a neighborhood of $e$ by a single variable $t$ such that

$$
\sigma=\mathrm{d} t
$$

is a left invariant form.

We next express the group product analytically. The product of the point with coordinate $s$ with that of coordinate $t$ will have coordinate $u$ given by

$$
u=p(s, t)
$$

with

$$
p(s, 0)=s, p(0, t)=t
$$

according to $x e=x, e y=y$. In coordinates

$$
L_{s}: t \rightarrow u=p(s, t)
$$

the left invariance of $\sigma, L_{s}^{*} \sigma=\sigma$, means

$$
\mathrm{d} t=\frac{\partial p}{\partial t} \mathrm{~d} t
$$

hence

$$
\frac{\partial p}{\partial t}=1, \quad p(s, t)=t+\varphi(s)
$$

setting $t=0$ :

$$
s=p(s, 0)=\varphi(s)
$$

and so

$$
p(s, t)=s+t
$$

Hence, $G$ is Abelian (commutative).

\subsection{The Action of Lie Groups on the Manifold}

Let $G$ denote a Lie group as the unit element of which is 1 and the Lie algebra of 
which is $g$. G-manifolds. Let $W$ be a smooth (i.e.: $C^{\infty}$ ) manifold on which $G$ acts by diffeomorphisms.

1) The action is written, that is, $G \times W \rightarrow W$,

$$
(g, x) \rightarrow g \cdot x
$$

As shown in Figure 7, the Lie Group $G$ represents a left action that is

$$
g \cdot(h \cdot x) \mapsto(g \cdot h) \cdot x \text { for all } g, h \in G, \quad x \in W .
$$

Here, a manifold endowed with a G-action is called is a G-manifold.

2) In physics, a Lie group often appears as the set of transformations acting on a Manifold. For example, $S O(3)$ special orthogonal group is the group of rotations in $\mathbb{R}^{3}$, while the Poincare's Group is the set of transformations acting on the Minkowski space-time. From Figure 8, it gives an idea about $S O(3)$ special orthogonal group is the group of rotations in $\mathbb{R}^{3}$ which identified by the $T$ transformations of Space time actions on a Manifold which appears a Lie group.

3) Flow is an action of $\mathbb{R}$ on a manifold $M$. If a flow is periodic with a period $T$, it may be regarded as an action of $U(1)$ real Orthogonal group or $S O(2)$ on $M$. In Figure 9. Given a periodic flow $\sigma(t, x)$ with period $T$ we construct a new action on $M$ is

$$
\sigma(\exp (2 \pi i t / T), x) \equiv \sigma(t, x) \text { whose group } G \text { is } U(1)
$$

4) Let $M \in G L(n, \mathbb{R})$ and let $x \in \mathbb{R}^{n}$. The action of $G L(n, \mathbb{R})$ on $\mathbb{R}^{n}$ is defined by the usual matrix action on a vector.

$$
\sigma:(M, x)=M \cdot x
$$

The action of the subgroups of the general linear group $G L(n, \mathbb{R})$ is defined similarly. They may also act in a smaller space. As shown in Figure 10, the two-dimensional surface of a (three-dimensional) ball in three-dimensional space is a 2 -sphere, often simply called a sphere as the $n-1$ dimensional boundary of a ( $n$-dimensional) $n$-ball is an $(n-1)$-sphere, it shows that, $O(n)$ orthogonal group acts on $S^{n-1}(r)$, an $(n-1)$ sphere of radius $r$ of orthogonal projection in space.

The action is written by,

$$
\sigma: O(n) \times S^{n-1}(r) \rightarrow s^{n-1}(r) .
$$

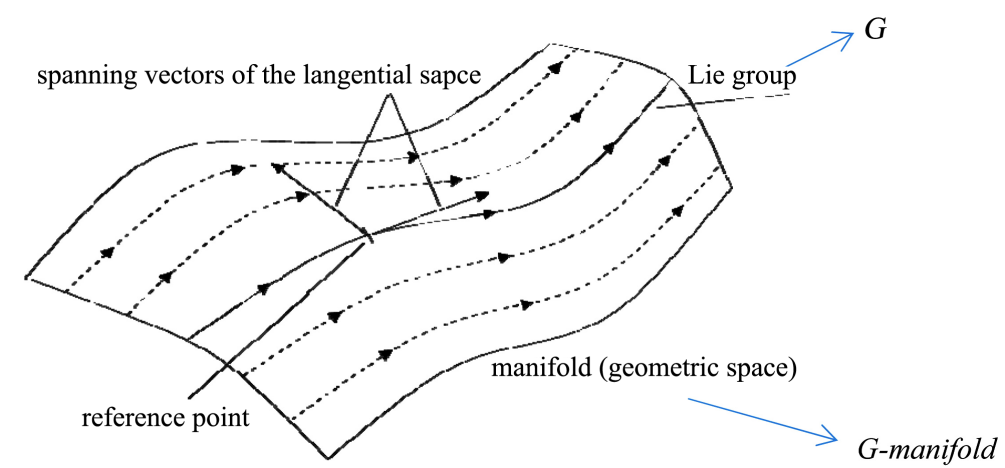

Figure 7. Lie group with manifolds. 


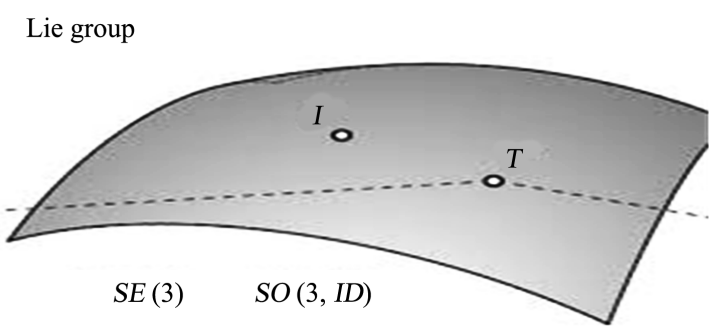

Figure 8. Lie group with $S O(3)$.

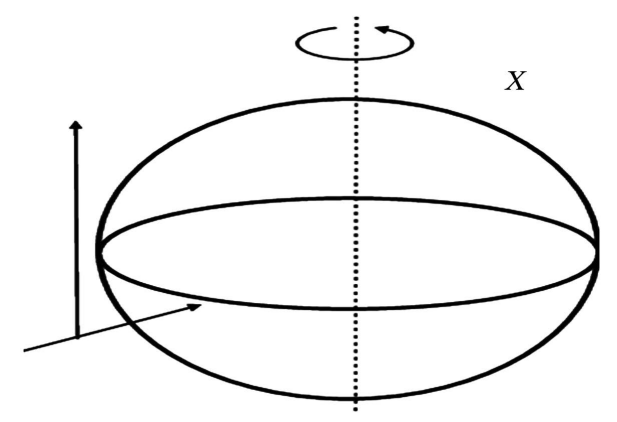

Figure 9. Lie group on $S O(2)$.

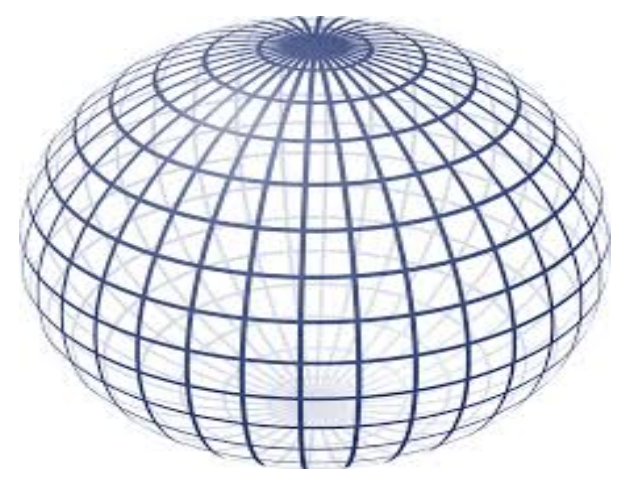

Figure 10. 2-Sphere of radius $r$.

\section{Conclusion and Recommendations}

\subsection{Conclusion}

Linear algebraic groups and Lie groups are two branches of group theory that have experienced advances and have become subject areas in their own right. From this paper, we have seen that Lie group embodies three different forms of mathematical structure. Firstly, it has a group structure. Secondly, the elements of this group also form a "topological space" so that it may be described as being a special case of "topological group". Finally, the elements also constitute an "analytic manifold". More generally Lie groups are differentiable manifolds which have very important consequences on Manifolds which are locally Euclidean spaces. We have summarized that by using differentiable structure, we can approximate the neighbourhood of any point of a Lie group by a Euclidean space which is the tangent space to the Lie group at that particular point. This approximation is some sort of local linearization of the Lie group. 


\subsection{Recommendations}

We would like to recommend that we have the plan to analyze these general operations and actions of the Lie group on manifolds for scientific research fields. Also, we plan to study more with Lie group and update their applications with lots of analyzing factors.

\section{Conflicts of Interest}

The authors declare no conflicts of interest regarding the publication of this paper.

\section{References}

[1] Nahm, W., Vladimir, R., and Manfred, S. (1976) The Classification of Graded Lie Algebras. Physics Letters B, 61, 383-384. https://doi.org/10.1016/0370-2693(76)90594-3

[2] Cahen, M. and Gutt, S. (1982) Regular* Representations of Lie Algebras. Letters in Mathematical Physics, 6, 395-404. https://doi.org/10.1007/BF00419321

[3] Gelfand, I.M., Graev, M.I. and Vertik, A.M. (1977) Representations of the Group of Smooth Mappings of a Manifold X into a Compact Lie Group. Compositio Mathematica, 35, 299-334.

[4] Albeverio, S. and Høegh-Krohn, R. (1978) The Energy Representation of Sobolev-Lie Groups. Compositio Mathematica, 36, 37-51.

[5] Celledoni, E. and Owren, B. (2003) Lie Group Methods for Rigid Body Dynamics and Time Integration on Manifolds. Computer Methods in Applied Mechanics and Engineering, 192, 421-438. https://doi.org/10.1016/S0045-7825(02)00520-0

[6] Gepner, D. and Witten, E. (1986) String Theory on Group Manifolds. Nuclear Physics B, 278, 493-549. https://doi.org/10.1016/0550-3213(86)90051-9

[7] Iserles, A. and Nørsett, S.P. (1999) On the Solution of Linear Differential Equations in Lie Groups. Philosophical Transactions of the Royal Society of London. Series A: Mathematical, Physical and Engineering Sciences, 357, 983-1019. https://doi.org/10.1098/rsta.1999.0362

[8] Goldman, W.M., and Millson, J.J. (1988) The Deformation Theory of Representations of Fundamental Groups of Compact Kähler Manifolds. Publications Mathématiques de IIHÉS, 67, 43-96. https://doi.org/10.1007/BF02699127

[9] Iserles, A., et al. (2000) Lie-Group Methods. Actanumerica, 9, 215-365. https://doi.org/10.1017/S0962492900002154

[10] Gallier, J. and Quaintance, J. (2012) Notes on Differential Geometry and Lie Groups. Department of Computer and Information Science, University of Pennsylvania Philadelphia, USA.

[11] Karl-Hermann, N. (2006) Towards a Lie Theory of Locally Convex Groups. Japanese Journal of Mathematics, 1, 291-468.

https://doi.org/10.1007/s11537-006-0606-y

[12] Ferreira, L.A. (2000) Lecture Notes in Lie Algebras and Lie Grups. IFT/UNESP. 